Revista Tecnologia e Ambiente, v. 21, 2014, Criciúma, Santa Catarina. ISSN: 1413-8131 (versão impressa) ISSN: 2358-9426 (versão eletrônica)

\title{
APIFAUNA (HYMENOPTERA: APIDAE) EM ÁREA DE RESTINGA NO SUL DE SANTA CATARINA, BRASIL
}

\author{
BEE SPECIES ASSEMBLY (HYMENOPTERA: APIDAE) IN A RESTINGA \\ AREA IN THE SOUTHERN REGION OF SANTA CATARINA, BRAZIL
}

\author{
Robson Siqueira Patricio ${ }^{1,2}$, Andressa da Silva Pereira ${ }^{1,2}$, Samuel Galvão Elias ${ }^{1,2}$, \\ Camila Brulezi Furlanetto², Mainara Figueiredo Cascaes², Alexandra Rocha Vinholes², \\ João Luis Osório Rosado², Birgit Harter-Marques²:3*
}

\begin{abstract}
Resumo
Inventários biológicos são importantes ferramentas para o conhecimento da biodiversidade e auxiliam no entendimento das alterações provenientes de processos antrópicos ou naturais. Inventários de comunidades de abelhas são especialmente relevantes e ainda escassos em ambiente de restinga na região sul do Estado de Santa Catarina. Nosso objetivo foi analisar a comunidade de abelhas em área de restinga arbórea no município de Araranguá, extremo sul catarinense. A amostragem de abelhas ocorreu sobre as espécies vegetais com flores, quinzenalmente, entre setembro de 2012 e agosto de 2013, em transectos pré-estabelecidos. No total, foram coletados 533 indivíduos distribuídos em 39 espécies e cinco subfamílias. Apinae e Halictinae apresentaram maior riqueza e abundância, corroborando outros inventários realizados no sul do Brasil, o que pode estar relacionado às diferentes organizações sociais e hábitos generalistas de forrageio das espécies. A riqueza de abelhas foi considerada baixa em comparação com outros estudos realizados no sul do Brasil, o que pode estar relacionado ao grau de fragmentação, pois ambientes fragmentados afetam negativamente as comunidades, podendo levá-las a extinção.
\end{abstract}

Palavras-chave: Diversidade de abelhas, restinga, Mata Atlântica.

\section{Abstract}

Biological inventories are important tools to gain knowledge about biodiversity, and to assist in understanding changes caused by anthropogenic or natural processes. Taxonomic inventories involving bee communities are especially relevant and scarce in coastal sand dune environments in the southern state of Santa Catarina. Our goal of this study was to examine the bee community in a wooded restinga area in the municipality of Araranguá at the southern end of Santa Catarina state. Sampling of bees occurred on all plant species with flowers, fortnightly, between September 2012 and August 2013, along predetermined transects. In total, 533 individuals of 39 bee species distributed in five subfamilies were collected. Apinae and Halictinae showed greatest richness and abundance, corroborating other inventories conducted in southern Brazil, which may be related to different social organizations and generalist foraging behavior of their species. The richness of bees was considered low compared with other studies conducted in

\footnotetext{
${ }^{1}$ Bolsista do Programa de Iniciação Científica (PIBIC e PIC-170), Universidade do Extremo Sul Catarinense (UNESC), Criciúma, Santa Catarina.

2 Laboratório de Interação Animal-Planta, Universidade do Extremo Sul Catarinense (UNESC), Criciúma, Santa Catarina.

3 Docente do Programa de Pós-Graduação em Ciências Ambientais (PPGCA), Universidade do Extremo Sul Catarinense (UNESC), Av. Universitária, Bairro Universitário, CEP 88806-000, Criciúma, Santa Catarina. E-mail: bhm@unesc.net
} 
southern Brazil, which could be related to the degree of fragmentation, because fragmented environments act negatively on communities, and may in the future lead them to extinction.

Keywords: Bee diversity, coastal sand dune, Atlantic Forest.

\section{Introdução}

Sucessivas transgressões e regressões das águas marinhas em direção ao continente e a sedimentação proveniente do próprio continente, tomaram a forma de extensos cordões litorâneos arenosos, onde hoje se encontra o litoral brasileiro, com cerca de $80 \%$ de extensão, sendo formado por restingas e dunas de areia (ARAÚJO; LACERDA, 1987; ALBERTONI; ESTEVES, 1999; PEREIRA et al., 2011). Após essa deposição de sedimentos arenosos, alguns fatores, como ventos e correntes litorâneas, passaram a modelar essa diversificada topografia, podendo apresentar complexos sistemas de corpos lagunares em algumas regiões (ARAÚJO; LACERDA, 1987; DORNELES; WAECHTER, 2004).

Segundo a Resolução nº 261/1999 do Conselho Nacional do Meio Ambiente (CONAMA) (BRASIL, 1999, p.1), entende-se restinga como:

[...] um conjunto de ecossistemas que compreende comunidades vegetais florísticas e fisionomicamente distintas, situadas em terrenos predominantemente arenosos, de origens marinha, fluvial, lagunar, eólica ou combinações destas, de idade quaternária, em geral com solos pouco desenvolvidos.

A vegetação de restinga exerce papel fundamental na preservação de espécies animais, migratórias ou não, servindo como recurso alimentar, abrigo e locais específicos para reprodução (BRASIL, 1999). Também auxilia na drenagem do solo nesses ambientes (FALKENBERG, 1999) e diminui os efeitos de transporte de areia causados pelo vento, que é um importante agente modificador da paisagem litorânea (CORDAZZO; SEELIGER, 1987).

Desde a chegada dos colonizadores, os ambientes costeiros vêm sendo destruídos, pelo fato de que a ocupação humana se concentrou por muito tempo nessas regiões (ARAÚJO; LACERDA, 1987; ALBERTONI; ESTEVES, 1999). Registros encontrados nos sambaquis indicam que essa ocupação é muito mais antiga e data cerca de 8 mil anos atrás (KNEIP, 1987). O desmatamento talvez seja a mais antiga pressão antrópica sofrida por esses ecossistemas, reduzindo-a em pequenas manchas distribuídas 
Revista Tecnologia e Ambiente, v. 21, 2014, Criciúma, Santa Catarina. ISSN: 1413-8131 (versão impressa) ISSN: 2358-9426 (versão eletrônica)

pelo litoral brasileiro (ARAÚJO; LACERDA, 1987; SIMINSKI; FANTINI, 2004; KORTE et al., 2013).

O Estado de Santa Catarina possui um litoral com $460 \mathrm{~km}$ de extensão, e apresenta faixas que podem ir de alguns metros até cerca de $7 \mathrm{~km}$ em direção ao continente (REITZ, 1961; SANTA CATARINA, 2013). No entanto, em alguns municípios, os ambientes naturais deram lugar a massivas construções e rodovias (KORTE et al., 2013). Essa grande expansão imobiliária, somada a outras atividades humanas como a mineração de areia, a visitação turística e a agricultura, causaram elevado impacto sobre estes ecossistemas (ARAÚJO; LACERDA, 1987; BENSUSAN, 2006; GASPARIN et al., 2011). Segundo Korte et al. (2013), no estado catarinense as áreas de restinga encontradas em bom estado de conservação são aquelas de difícil acesso, ou que já estão incluídas em uma Unidade de Conservação.

Inventários biológicos são importantes ferramentas na obtenção de conhecimento sobre a biodiversidade de uma determinada região, além disso auxiliam no entendimento das alterações provenientes de processos antrópicos ou naturais (LEWINSOHN et al., 2001). Os polinizadores podem ser utilizados como organismos bioindicadores em muitos ambientes (KEVAN, 1999). Em comunidades tropicais a porcentagem de plantas polinizadas por animais chega a cerca de $90 \%$, sendo superior as zonas temperadas (OLLERTON et al., 2011). Apesar de moscas, borboletas e besouros serem citados como grupos de polinizadores para certas espécies de plantas, as abelhas são consideradas os principais insetos polinizadores da maioria das espécies de plantas nativas e agrícolas, pois estes organismos dependem do pólen como fonte protéica para o desenvolvimento da sua prole (BUZZI, 2005; MICHENER, 2007; RAVEN et al., 2007).

São conhecidas cerca de 18.000 espécies de abelhas no mundo, mas acredita-se que o número total possa chegar a 30.000 (MICHENER, 2007). No Brasil, estão descritas 1.678 espécies de abelhas (MOURE, 2007), sendo estimada uma riqueza de aproximadamente 3.000 espécies. A fauna brasileira apresenta cinco das sete subfamílias existentes no mundo, sendo elas Andreninae, Apinae, Colletinae, Halictinae e Megachilinae (SILVEIRA et al., 2002; MELO; GONÇALVES, 2005).

Alguns trabalhos relacionados a comunidades de abelhas em ambientes de restinga para os estados brasileiros foram realizados por Gottsberger et al. (1988), 
Albuquerque et al. (2007), Oliveira et al., (2010) no estado do Maranhão, Madeira-daSilva e Martins (2003) na Paraíba, Viana (1999), Viana e Kleinert (2005) na Bahia, Zanella et al. (1998) no Paraná e Alves-dos-Santos (1999) no estado do Rio Grande do Sul.

Em Santa Catarina, estudos envolvendo a fauna apícola em ambientes costeiros são escassos, podendo-se citar os trabalhos de Mouga (2004) em São Bento do Sul, Steiner et al. (2006) em Florianópolis e Kamke et al. (2011) em Palhoça. Com base na importância dos inventários taxonômicos que envolvem comunidades de abelhas, e sua escassez em ambiente de restinga na região sul do estado, o presente estudo buscou inventariar e analisar a comunidade de abelhas em uma área de restinga arbórea localizada no município de Araranguá, no extremo sul de Santa Catarina, assim como correlacionar a riqueza com as médias mensais de temperatura e pluviosidade.

\section{Material e Métodos}

\section{a) Área de estudo}

O presente estudo foi realizado em uma área de Restinga localizada nos limites dos municípios de Araranguá e Balneário Arroio do Silva entre as coordenadas UTM 22J 655197E/ 6797118S e 655696E/ 6797523S, no extremo sul de Santa Catarina (Figura 1). A área possui cerca de 18 hectares e sua altitude varia entre 8 e 39 metros em relação ao nível do mar (dados obtidos por aparelho de GPS), sendo que sua distância em relação ao mesmo é de aproximadamente $3 \mathrm{~km}$.

Nas áreas de entorno há presença de campo destinado a pastagem de gado, mineração de areia (a uma distância de 100 metros da área de estudo) e monocultura de Eucalyptus spp., onde foram introduzidas 75 caixas de Apis mellifera Linnaeus, 1758 alguns meses antes do início do presente estudo (comunicação pessoal, 2013). 
Revista Tecnologia e Ambiente, v. 21, 2014, Criciúma, Santa Catarina. ISSN: 1413-8131 (versão impressa) ISSN: 2358-9426 (versão eletrônica)

Figura 1. A) Localização geográfica dos municípios de Araranguá e Balneário Arroio do Silva; B) Localização da área de estudo, com destaque em amarelo para o local de coleta, compreendido entre as coordenadas UTM 22J 655197E/ 6797118S e 655696E/ 6797523S.
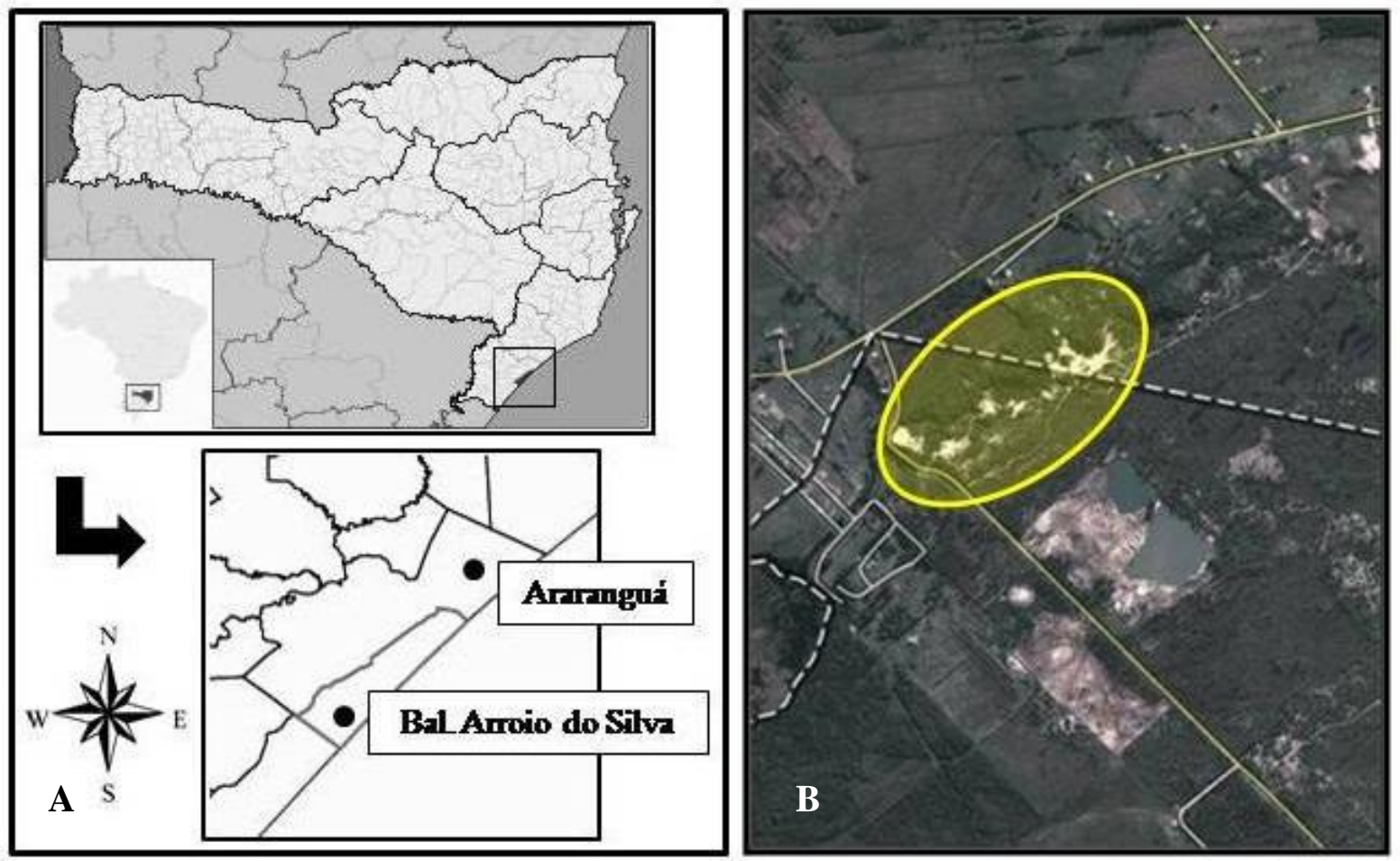

Fonte: Modificado de Google Earth (2014) e IBGE (2014)

O clima da região enquadra-se no tipo Cfa, clima subtropical úmido, com verões quentes e sem estação seca definida (EPAGRI, 2001). No período do presente estudo (setembro de 2012 e agosto de 2013), o valor total de pluviosidade (1.640 $\mathrm{mm}$ ) apresentou-se semelhante ao dos últimos 19 anos. A distribuição das chuvas foi muito irregular durante o ano, apresentando dois picos, o primeiro de janeiro a março e o segundo no mês de agosto. A temperatura média durante o estudo foi de $19,8{ }^{\circ} \mathrm{C}$, também sendo considerada semelhante em relação às médias normais da região.

A área encontra-se sob dunas móveis e dunas fixadas pela vegetação de restinga, apresentando um solo bem drenado e classificado como Neossolo, formado a partir de areias quartzosas de origem marinha (EPAGRI, 2001).

Quanto à vegetação, a classificação aqui adotada obedece os parâmetros estabelecidos na Resolução no 261, de 30 de junho de 1999 do CONAMA (BRASIL, 1999), que servem para caracterizar a vegetação de restinga quanto sua fitofisionomia e estágio sucessional no estado de Santa Catarina. 
A cobertura vegetal do presente estudo foi definida como Restinga Arbórea ou Mata de Restinga em estágio avançado de regeneração, onde a vegetação possui predomínio de espécies arbóreas com altura variando de 6 a 10 metros, podendo alcançar até 15 metros. Dentre as espécies arbóreas destacam-se: Clusia criuva Cambess. (Clusiaceae), Guapira opposita (Vell.) Reitz (Nyctaginaceae) e Ocotea pulchella (Nees \& Mart.) Mez (Lauraceae). Há também presença das epífitas: Tillandsia aeranthos Desf. ex Steud. e Aechmea nudicaulis (L.) Griseb. (Bromeliaceae) e um subbosque presente, com serapilheira acumulada e abundante (Figura 2).

Figura 2. Estágio avançado de regeneração da área de restinga arbórea, localizada no município de Araranguá (UTM 22J 655596E/ 6797390S) no extremo sul de Santa Catarina.

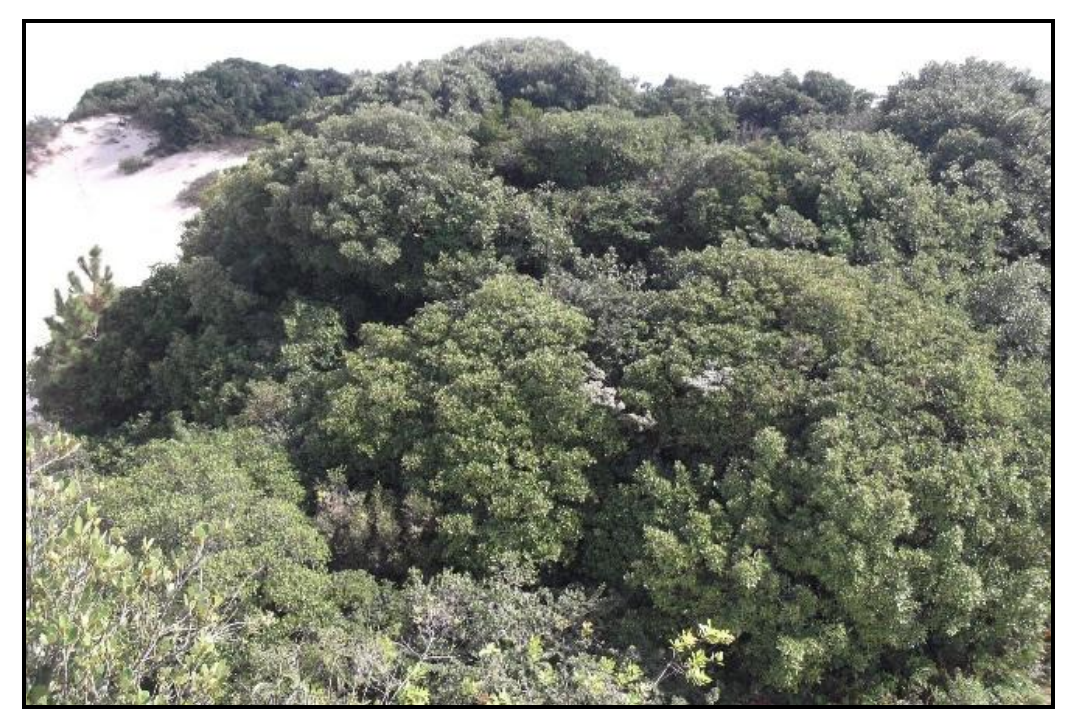

\section{b) Coleta das abelhas}

A metodologia de coleta das abelhas foi adaptada de Sakagami et al. (1967). As coletas foram realizadas quinzenalmente, em um único dia no período das $8 \mathrm{~h}$ às $16 \mathrm{~h}$, durante os meses de setembro de 2012 e agosto de 2013. Nos meses de outubro de 2012 e fevereiro de 2013 as coletas foram feitas mensalmente, devido a problemas logísticos. Desta forma, foram realizadas 22 coletas, totalizando 176 horas amostrais. Cada coleta foi realizada através de transectos preestabelecidos, que foram percorridos ao passo lento. No primeiro indivíduo de cada espécie vegetal encontrado em floração, as abelhas foram coletadas individualmente ou em grupo quando pousavam nas flores, com auxílio de rede entomológica durante 10 minutos. Para a coleta nas copas das árvores 
Revista Tecnologia e Ambiente, v. 21, 2014, Criciúma, Santa Catarina. ISSN: 1413-8131 (versão impressa) ISSN: 2358-9426 (versão eletrônica)

encontradas nas bordas, foi utilizada uma rede de cabo regulável, com alcance de até 9m de altura.

As abelhas coletadas foram colocadas em câmaras mortíferas com acetato de etila, e devidamente etiquetadas com número da planta visitada, o horário e a data de coleta.

Em laboratório, os espécimes foram transfixados e separados por morfoespécies. Posteriormente, os indivíduos foram identificados com auxílio de chaves genéricas (SILVEIRA et al., 2002), chaves específicas, através da consulta de especialistas, além da comparação com o material presente na coleção de referência do Laboratório de Interação Animal-Planta (LIAP) da Universidade do Extremo Sul Catarinense, onde foram depositados os exemplares coletados. A classificação adotada foi a de Melo e Gonçalves (2005).

\section{c) Análise de dados}

As abelhas associadas às plantas foram analisadas quantitativamente $\mathrm{e}$ qualitativamente através de listagens, análise da riqueza (S) e abundância $(\mathrm{N})$ das espécies. Foram calculados os índices de Shannon-Wiener ( $\left.\mathrm{H}^{\prime}\right)$ e de equitabilidade (J), utilizando-se o programa PAST 3.01 (HAMMER et al., 2001).

Para verificar a normalidade dos dados foi realizado o teste de Shapiro-Wilk e, posteriormente, utilizou-se o coeficiente de correlação de Pearson (r) através do programa SPSS 20.0 (IBM, 2011) para os cálculos de correlação entre a riqueza de abelhas e as médias mensais de temperatura e pluviosidade.

A curva do coletor foi construída, utilizando-se a acumulação de espécies durante o período de coleta. Para a determinação da suficiência amostral da comunidade foram utilizados os estimadores de riqueza Jacknife 1 e 2, Chao 1 e 2 e Bootstrap, bem como as curvas de rarefação das espécies em Mao Tau, com aleatorização de 50 vezes, utilizando-se o programa EstimatesS 8.2.0 (COLWELL, 2006).

Para cada espécie de abelha coletada foi determinada a medida faunística da constância pela equação: $C=\frac{(p \times 100)}{N}$, apresentada em Silveira Neto et al. (1976), onde: $\mathbf{C}=$ constância em percentual, $\mathbf{p}=$ número de coletas contendo a espécie em estudo, $\mathbf{N}=$ número total de coletas efetuadas. Assim, as espécies foram classificadas em constantes 
Revista Tecnologia e Ambiente, v. 21, 2014, Criciúma, Santa Catarina. ISSN: 1413-8131 (versão impressa) ISSN: 2358-9426 (versão eletrônica)

(quando presentes em mais de 50\% das coletas), acessórias (as encontradas entre $25 \%$ e $50 \%$ das coletas) ou acidentais (presentes em menos de $25 \%$ das coletas).

A dominância das espécies de abelhas encontradas na área de estudo foi determinada através do cálculo do limite de dominância a partir da equação $L D=\left(\frac{1}{s}\right) \times 100$, citado por Sakagami e Laroca (1971), onde: $\mathbf{L D}=$ representa o limite de dominância, $\mathbf{S}$ = representa o número total de espécies. Este parâmetro classifica as espécies em dominantes quando os valores do limite inferior de cada espécie apresentam-se superiores ao limite de dominância, e não dominantes quando os valores encontrados foram menores (adaptado de SILVA, 2005). O limite inferior de cada espécie foi calculado pela fórmula: $L i=\left(1-\frac{\left(k^{b} 1 x F\right)}{\left(k^{\prime} 2+\left(k^{\prime} 1 x F\right)\right)}\right) x 100$, onde: $\mathbf{L i}=$ limite inferior para cada espécie, $\mathbf{F}_{\mathbf{0}}=$ valor obtido da tabela de distribuição de $\mathrm{F}$ ao nível de 5\% de significância para graus de liberdade obtidos em k'1 e k'2 (ZAR, 1999), k'1= $2 x(N-n i+1), \mathbf{k}^{\prime} \mathbf{2}=2 x(n i+1), \mathbf{N}=$ número total de indivíduos, $\mathbf{n i}=$ número total de indivíduos da espécie i.

\section{Resultados}

Foi coletado um total de 533 abelhas (400 fêmeas e 133 machos), distribuídas em 39 espécies, 27 gêneros, 12 tribos e cinco subfamílias. A proporção entre o número de fêmeas e machos ficou em três fêmeas por cada macho coletado. Excluindo as operárias de Apis mellifera há redução desta proporção para 1,4 fêmeas por macho (Tabela 1).

Tabela 1. Abundância de abelhas coletadas em área de restinga arbórea no extremo sul de Santa Catarina, entre os meses de Setembro de 2012 e Agosto de 2013. (M) = machos; $(\mathrm{F})=$ fêmeas; $(*)=$ abelhas cleptoparasitas.

\begin{tabular}{|c|c|c|c|}
\hline \multirow{2}{*}{ Subfamília/ Tribo/ Espécie } & \multicolumn{3}{|c|}{ Número de indivíduos } \\
\hline & M & $\mathbf{F}$ & Total \\
\hline \multicolumn{4}{|l|}{ Andreninae } \\
\hline \multicolumn{4}{|l|}{ Panurgini } \\
\hline $\begin{array}{l}\text { Callonychium (Callonychium) petuniae Cure \& Wittmann, } \\
1990\end{array}$ & 21 & 20 & 41 \\
\hline \multicolumn{4}{|l|}{ Apinae } \\
\hline \multicolumn{4}{|l|}{ Apini } \\
\hline Apis mellifera Linnaeus, 1758 & 0 & 218 & 218 \\
\hline Bombus (Fervidobombus) morio(Swederus, 1787) & 0 & 2 & 2 \\
\hline
\end{tabular}




\begin{tabular}{|c|c|c|c|}
\hline \multirow{2}{*}{ Subfamília/ Tribo/ Espécie } & \multicolumn{3}{|c|}{ Número de indivíduos } \\
\hline & $\mathbf{M}$ & $\mathbf{F}$ & Total \\
\hline Eufriesea violacea (Blanchard, 1840) & 0 & 2 & 2 \\
\hline \multicolumn{4}{|l|}{ Centridini } \\
\hline Centris (Trachina) proxima (Blanchard, 1840) & 0 & 1 & 1 \\
\hline Centris (Hemisiella) tarsata Smith, 1874 & 0 & 3 & 3 \\
\hline Centris (Centris) decolorata Lepeletier, 1841 & 3 & 0 & 3 \\
\hline Epicharis (Anepicharis) chrysophyga (Friese, 1900) & 0 & 6 & 6 \\
\hline Epicharis (Anepicharis) dejeanii Lepeletier, 1841 & 0 & 2 & 2 \\
\hline \multicolumn{4}{|l|}{ Emphorini } \\
\hline Alepidoscelis imitatrix (Schrottky, 1909) & 2 & 0 & 2 \\
\hline Melitoma segmentaria (Fabricius, 1804) & 1 & 0 & 1 \\
\hline \multicolumn{4}{|l|}{ Ericrocidini } \\
\hline Mesoplia (Mesoplia) simillima Schrottky, 1920 * & 1 & 1 & 2 \\
\hline \multicolumn{4}{|l|}{ Eucerini } \\
\hline Melissoptila paraguayensis (Brèthes, 1909) & 2 & 0 & 2 \\
\hline Melissoptila setigera Urban, 1998 & 0 & 3 & 3 \\
\hline \multicolumn{4}{|l|}{ Xylocopini } \\
\hline Ceratina (Crewella) assuncionis Strand, 1910 & 1 & 1 & 2 \\
\hline Ceratina sp.1 & 3 & 6 & 9 \\
\hline Xylocopa (Stenoxylocopa) artifex Smith, 1874 & 0 & 1 & 1 \\
\hline Xylocopa (Neoxylocopa) brasilianorum (Linnaeus, 1767) & 0 & 11 & 11 \\
\hline \multicolumn{4}{|l|}{ Colletinae } \\
\hline \multicolumn{4}{|l|}{ Colletini } \\
\hline Colletes rugicolis Friese, 1900 & 1 & 1 & 2 \\
\hline \multicolumn{4}{|l|}{ Diphaglossini } \\
\hline Ptiloglossa virgilii (Friese, 1900) & 1 & 0 & 1 \\
\hline \multicolumn{4}{|l|}{ Paracolletini } \\
\hline Cephalocolletes rugata Urban, 1995 & 1 & 1 & 2 \\
\hline Hexantheda eneomera Urban \& Graf, 2000 & 2 & 2 & 4 \\
\hline Sarocolletes guaritarum Urban 1995 & 2 & 1 & 3 \\
\hline Tetraglossula bigamica (Strand, 1910) & 76 & 5 & 81 \\
\hline \multicolumn{4}{|l|}{ Halictinae } \\
\hline \multicolumn{4}{|l|}{ Augochlorini } \\
\hline Augochlora (Augochlora) amphitrite (Schrottky, 1910) & 0 & 11 & 11 \\
\hline Augochlorela ephyra (Schrottky, 1910) & 1 & 2 & 3 \\
\hline Augochloropsis discors (Vachal, 1903) & 0 & 4 & 4 \\
\hline Augochloropsis semele (Schrottky, 1902) & 1 & 8 & 9 \\
\hline Augochloropsis sp.1 & 0 & 14 & 14 \\
\hline Augochloropsis sp.2 & 0 & 1 & 1 \\
\hline Pseudaugochlora graminea (Fabricius, 1804) & 1 & 9 & 10 \\
\hline Tectochlora hamata Gonçalves \& Melo, 2006 & 5 & 43 & 48 \\
\hline
\end{tabular}


Subfamília/ Tribo/ Espécie

Número de indivíduos

\section{Halictini}

Dialictus sp. 1

Dialictus sp. 2

Pseudagapostemon (Pseudagapostemon) pruinosus Moure

\& Sakagami, 1984

\section{Megachilinae}

Megachilini

Coelioxys (Glyptocoelioxys) chacoensis Holmberg, 1903* $\quad 1 \quad 1 \quad 1 \quad 2$ $1903 *$

Coelioxys (Glyptocoelioxys) cerasiopleura Holmberg,

\begin{tabular}{llccc}
$1903 *$ & & 0 & 1 & 1 \\
& Coelioxys sp.1* & 1 & 0 & 1 \\
& Megachile (Pseudocentron) terrestris Schrottky, 1902 & 1 & 2 & 3 \\
\hline Total & 134 & 399 & 533 \\
\hline
\end{tabular}

Apinae apresentou um número maior de espécies $(\mathrm{S}=17)$ e de indivíduos $(\mathrm{n}=$ 270) em relação as demais subfamílias, seguida por Halictinae $(S=10$ e $n=122)$, Colletinae $(S=6$ e $n=93)$, Megachilinae $(S=3$ e $n=7)$ e Andreninae $(S=1$ e $n=41)$

(Figuras 3 e 4) e o índice de diversidade de Shannon-Wiener (H') foi de 2,3 e a equitabilidade (J) 0,628.

Figura 3. Distribuição da riqueza de abelhas nas cinco subfamílias coletadas em um ambiente de restinga no extremo sul de Santa Catarina.

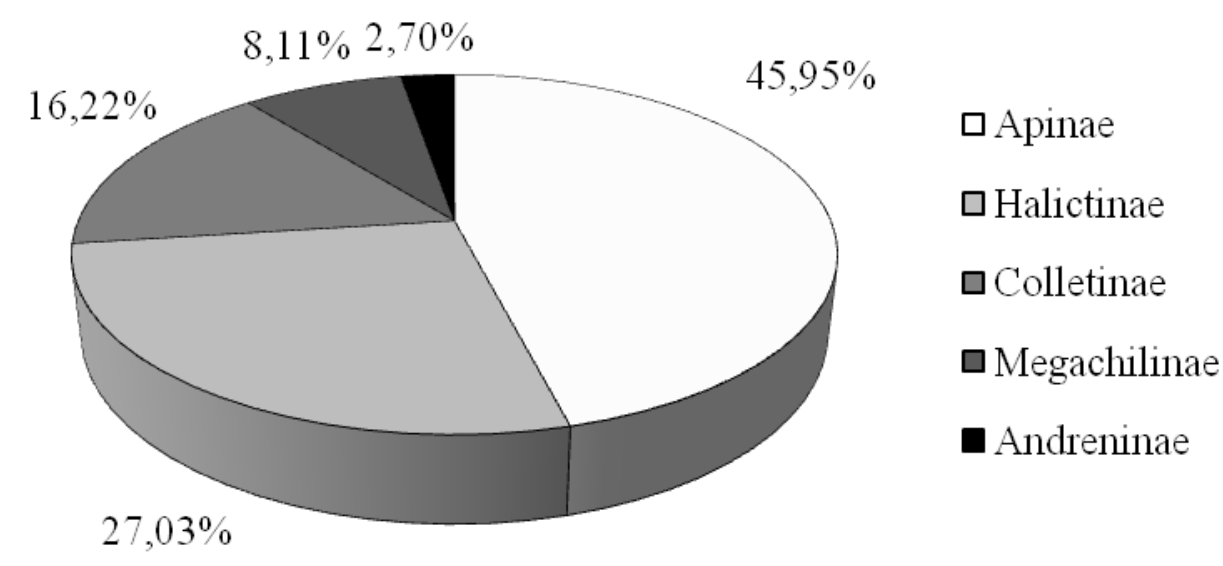


Figura 4. Distribuição da abundância de abelhas nas cinco subfamílias coletadas em um ambiente de restinga no extremo sul de Santa Catarina.

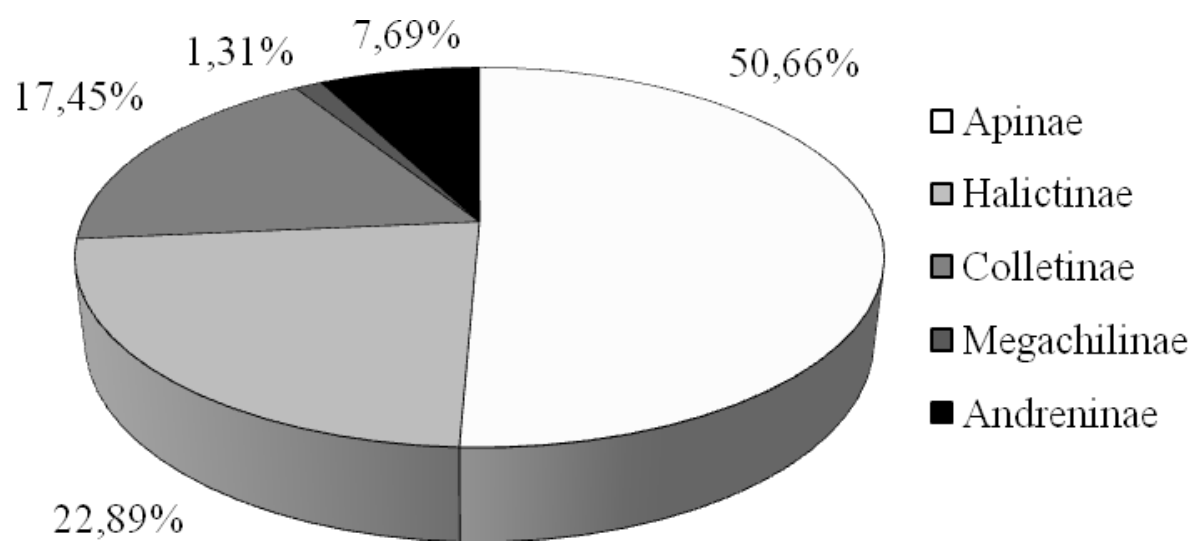

Do total das espécies amostradas, as mais abundantes foram Apis mellifera $(\mathrm{n}=$ 213), Tetraglossula bigamica $(\mathrm{n}=81)$, Tectochlora hamata $(\mathrm{n}=48)$, Callonychium petuniae $(\mathrm{n}=41)$ e Augochloropsis sp.1 ( $\mathrm{n}=14)$, totalizando $74,48 \%$ dos indivíduos amostrados. Não foram encontradas espécies de abelhas eussociais nativas (Meliponina).

A curva de rarefação mostrou tendência à estabilização. Os valores mínimos e máximos calculados pelos estimadores de riqueza ficaram entre Chao $1(40,9)$ e Jacknife $2(50,9)$, respectivamente, sugerindo que entre 76,6 e 95\% da fauna de abelhas presentes no local foram efetivamente amostradas, sendo a amostragem considerada suficiente (Figura 5). 
Figura 5. Curva de rarefação e a média efetiva (Mao Tau) das espécies de abelhas coletadas em um ambiente de restinga no extremo sul de Santa Catarina.

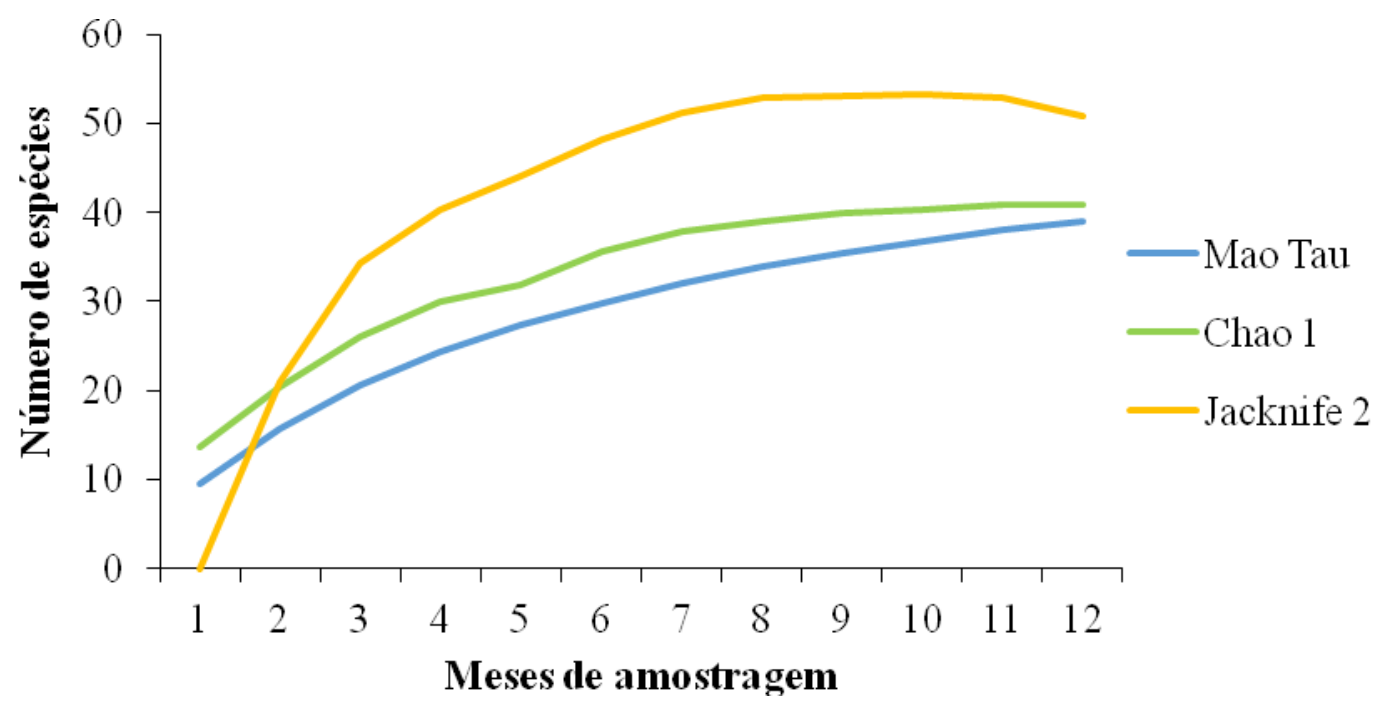

De todas as espécies, apenas duas foram consideradas constantes (Apis mellifera e Pseudaugochlora graminea), 13 acessórias (Augochlora amphitrite, Augochloropsis discors, Augochloropsis semele, Augochloropsis sp.1, Callonychium petuniae, Ceratina sp.1, Dialictus sp.1, Dialictus sp.2, Hexantheda eneomera, Pseudagapostemon pruinosus, Tectochlora hamata, Tetraglossula bigamica e Xylocopa brasilianorum) e 24 acidentais.

As espécies cleptoparasitas foram representadas por um total de seis indivíduos, quatro deles pertencentes a três espécies do gênero Coelioxys, sendo que uma delas foi identificada somente ao nível de gênero, e dois indivíduos de Mesoplia simillima. Juntas essas quatro espécies cleptoparasitas perfizeram aproximadamente $10 \%$ do total de espécies coletadas no presente estudo.

O limite de dominância (Ld) foi de 2,56, sendo que quatro espécies apresentaram um limite inferior calculado $(\mathrm{Li})$ superior e foram consideradas como dominantes: Apis mellifera $(\mathrm{Li}=38,44)$; Tetraglossula bigamica $(\mathrm{Li}=13,01)$; Tectochlora hamata $(\mathrm{Li}=7,30)$; e Callonychium petuniae $(\mathrm{Li}=6,06)$. Estas espécies somam $72,8 \%$ dos espécimes amostrados.

A comunidade de abelhas apresentou variação em relação à riqueza de visitantes durante os meses do ano, sendo registrado o maior número de espécies no período mais quente do ano (entre novembro de 2012 a março de 2013). Neste período, o número de 
espécies de abelhas visitando as flores variou entre 14 e 21 espécies, com um pico observado em janeiro de 2013.

Nos meses mais frios do ano (junho e julho de 2013), onde as médias de temperatura não ultrapassaram os $16^{\circ} \mathrm{C}$, foram amostradas apenas duas espécies de abelhas nas flores. A riqueza apresentou correlação positiva em relação às médias mensais de temperatura $\left(\mathrm{r}^{2}=0,914 ; \mathrm{p}<0,01\right)$ (Figura 6), no entanto, não houve correlação com a pluviosidade $\left(r^{2}=-0,205 ; p>0,05\right)$.

Figura 6. Riqueza de abelhas amostradas na área de estudo em relação às médias de temperatura entre os meses de setembro de 2012 a agosto de 2013.

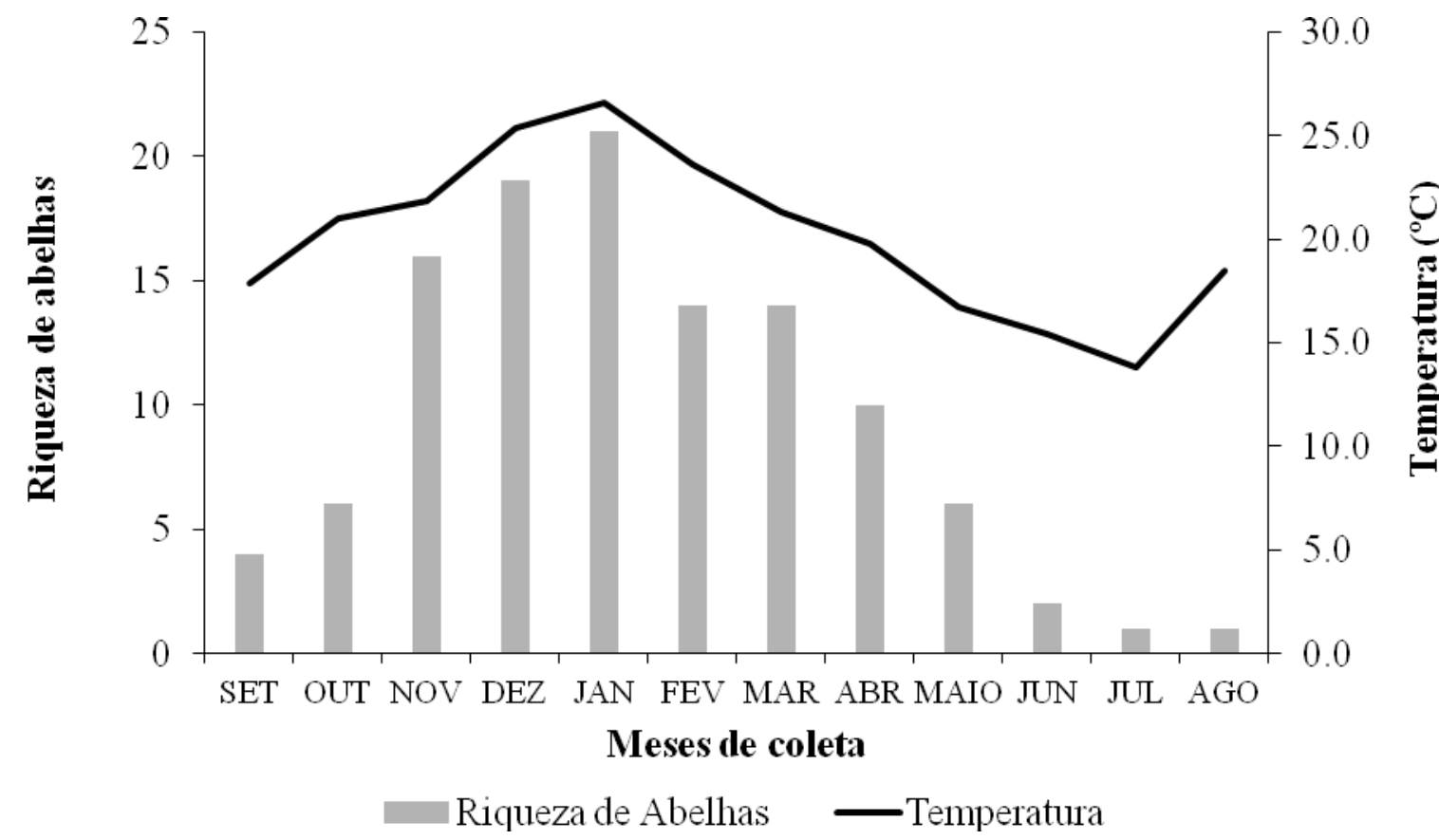

\section{Discussão}

Todas as cinco subfamílias de abelhas com ocorrência no Brasil (SILVEIRA et al., 2002) foram amostradas no presente estudo e seguiram a seguinte ordem decrescente de riqueza: Apinae > Halicitinae > Colletinae > Megachilinae > Andreninae.

As abelhas da região neotropical seguem um gradiente latitudinal onde, mais ao sul Andreninae, Colletinae e Megachilinae apresentam menor riqueza de espécies em comparação a Apinae e Halictinae (ROUBICK, 1989). A maior riqueza concentrada em 
Revista Tecnologia e Ambiente, v. 21, 2014, Criciúma, Santa Catarina. ISSN: 1413-8131 (versão impressa) ISSN: 2358-9426 (versão eletrônica)

Apinae e Halictinae também foi encontrada em diversos inventários realizados na região sul do Brasil (WITTMANN; HOFMANN, 1989; ALVES-DOS-SANTOS, 1999; HARTER, 1999; MOUGA, 2004; GONÇALVES; MELO, 2005; SILVA, 2005; KRUG, 2007; TRUYLIO; HARTER-MARQUES, 2007; CASCAES, 2008; BEZ, 2009; ROSA, 2011; MOUGA et al., 2012).

O fato de Apinae e Halictinae apresentarem alta representatividade no presente estudo e nos inventários supracitados pode estar relacionado à maior variedade morfológica, diferentes organizações sociais, variando desde espécies solitárias até altamente sociais, e hábitos generalistas de forrageio de suas espécies (ROUBICK, 1989; ENGEL, 2000; MICHENER, 2007).

Augochlorini apresentou mais espécies em relação às demais tribos, estas espécies são multivoltinas, o que explica o fato de seus indivíduos serem coletados durante diferentes épocas do ano (SCHLINDWEIN, 1998; ALVES-DOS-SANTOS, 1999). A diversidade de Augochlorini aumenta em regiões mais ao sul do Brasil, sendo que algumas de suas espécies podem formam agregações de ninhos com muitos indivíduos (ROUBICK, 1989; ENGEL, 2000; MICHENER, 2007). Esta característica pode explicar por que Halictinae foi a segunda subfamília mais representativa em número de espécies neste estudo.

A espécie Tectochlora hamata foi uma das espécies dominantes. Em outros estudos, a espécie é descrita como sendo específica de áreas de dunas, sendo encontrada com alta abundância (KAMKE et al., 2011; GONÇALVES; MELO, 2005). Em Santa Catarina, esta espécie havia sido coletada apenas na região da Grande Florianópolis (STEINER, et al., 2006; KAMKE et al., 2011), evidenciando a importância da realização de inventários abrangendo diferentes regiões.

As tribos Centridini, Emphorini, Ericrocidini, Eucerini e Xylocopini, que fazem parte dos Apinae não corbiculados (MELO; GONÇALVES, 2005), apresentaram abelhas com tamanho corporal de médio a grande porte. Apesar de que seus valores de dominância e constância se mostraram baixos, o que pode estar associado aos hábitos solitários, à ocorrência rara ou à sazonalidade de suas espécies, essas cinco tribos representaram cerca de $40 \%$ do total de espécies amostradas. Algumas espécies das tribos Centridini, e Emphorini estão mais relacionadas a ambientes abertos (VIANA; ALVES-DOS-SANTOS, 2002). Ambientes com incidência de ventos mais forte, como 
Revista Tecnologia e Ambiente, v. 21, 2014, Criciúma, Santa Catarina. ISSN: 1413-8131 (versão impressa) ISSN: 2358-9426 (versão eletrônica)

no caso de áreas do litoral, podem influenciar no predomínio de abelhas com tamanho grande (GOTTSBERGER et al. 1988).

A ausência de operárias das espécies de Meliponini no presente estudo pode estar relacionada à escassez de locais apropriados para nidificação, visto que a maioria de suas espécies nidifica em troncos ocos pré-existentes de árvores e, ao forragear, podem alcançar distâncias entre 500 a 3.000 metros (NOGUEIRA NETO, 1997). Desta forma, a presença destas espécies em uma área é diretamente influenciada pela qualidade e proximidade de fragmentos florestais, que oferecem condições favoráveis para seu estabelecimento (ROUBICK, 1989; SILVEIRA et al., 2002; MARTINS et al., 2004).

O índice de Shannon-Wiener foi superior a outros inventários realizados em Santa Catarina, onde a riqueza total de abelhas foi superior ou próxima a 100 espécies (SILVA, 2005; KRUG, 2007; TRUYLIO; HARTER-MARQUES, 2007), porém, a equitabilidade foi relativamente baixa. Um fato que pode explicar este resultado é uma maior abundância concentrada em poucas espécies, enquanto muitas espécies apresentaram número reduzido de indivíduos. Isso se confirmou ao se analisar o índice de Shannon-Wiener, excluindo a espécie A. mellifera, resultando em uma equitabilidade aumentada $(\mathrm{J}=0,756)$.

A. mellifera representou cerca de $40 \%$ do total de abelhas coletadas e foi registrada em quase todos os meses do ano nas flores das espécies melitófilas. Estes resultados expressam a proximidade do ambiente estudado com a atividade de apicultura praticada no seu entorno e corroboram outros inventários, onde a espécie tem apresentado maior número de indivíduos em relação às espécies nativas (ALVES-DOSSANTOS, 1999; TRUYLIO; HARTER- MARQUES, 2007; CASCAES, 2008; BEZ, 2009; ROSA, 2011; MOUGA et al., 2012). Os maiores valores de abundância, constância e dominância encontrados nesta espécie deve-se ao fato de que suas colônias possuem elevado número de indivíduos, maior amplitude trófica (WILMS et al., 1996) e eficiente capacidade de comunicação entre suas operárias na busca por fontes de alimento (ROUBICK, 1989). Além disso, ela é mais tolerante do que as espécies nativas menores em relação às baixas temperaturas para forrageio (MENEZES et al., 2007).

Entre todas as espécies de abelhas coletadas no presente estudo, quatro espécies são cleptoparasitas, representando cerca de $10 \%$ da comunidade. São elas: Mesoplia 
Revista Tecnologia e Ambiente, v. 21, 2014, Criciúma, Santa Catarina. ISSN: 1413-8131 (versão impressa) ISSN: 2358-9426 (versão eletrônica)

simillima, Coelioxys chacoensis, Coelioxys cerasiopleura, Coelioxys sp.1. As espécies do gênero Mesoplia parasitam os ninhos de espécies dos gêneros Centris e Epicharis. Já as espécies de Coelioxys parasitam os ninhos de espécies de Megachile, podendo também ocupar ninhos de Centris e Euglossa (SILVEIRA et al., 2002; MICHENER, 2007). Com exceção de Euglossa, os demais gêneros acima citados foram registrados na área do estudo, o que explicaria a presença destas espécies cleptoparasitas.

O período de maior atividade das abelhas encontrado durante os meses mais quentes do ano corrobora muitos estudos realizados na região sul brasileira, onde a riqueza e abundância de abelhas coletadas é maior nos meses da primavera e verão (ALVES-DOS-SANTOS, 1999; KAMKE et al., 2011). Isso se explica pelo fato de que na região sul muitas espécies de abelhas se mantém inativas durante os meses mais frios do ano (ALVES-DOS-SANTOS, 1999; TRUYLIO; HARTER-MARQUES, 2007; KRUG; ALVES-DOS-SANTOS, 2008; KAMKE et al., 2011).

Quando comparada a outros estudos realizados em ambientes de restinga no sul do Brasil, a riqueza de abelhas aqui encontrada foi considerada baixa (ALVES-DOSSANTOS, 1996; ZANELLA et al., 1998; MOUGA, 2004; STEINER et al., 2006; KAMKE et al., 2011), sendo maior ou semelhante a muitos outros realizados na região nordeste do país (VIANA, 1999; MADEIRA-DA-SILVA; MARTINS, 2003; VIANA; KLEINERT, 2005; OLIVEIRA et al., 2010). Segundo Silveira, Melo e Almeida (2002) e, alguns resultados são difíceis de serem comparados, devido a diferenças nas metodologias aplicadas nos inventários. No sul do Brasil, os inventários realizados em longos períodos (ORTOLAN, 1989; HARTER, 1999) ou em formações vegetais variadas (WITTMANN; HOFMANN, 1989; ALVES-DOS-SANTOS, 1996) resultaram em extensas listas taxonômicas, corroborando Ricklefs (2010) que confirma que em comunidades biológicas, o aumento do tamanho amostral resulta em um incremento no número de espécies. Entretanto, no presente estudo, onde a comunidade das abelhas foi coletada apenas durante um ano, os estimadores de riqueza Chao 1 e Jacknife 1 apontaram que entre 80,0 e $97,8 \%$ da fauna de abelhas presentes no local foram efetivamente amostradas, sendo a amostragem foi considerada suficiente. Desta forma, acredita-se que a riqueza das abelhas não aumentará consideravelmente com um maior esforço amostral e que o número de espécies coletadas representa a situação real da área estudada. Assim sendo, a baixa riqueza encontrada pode estar relacionado ao grau de 
Revista Tecnologia e Ambiente, v. 21, 2014, Criciúma, Santa Catarina. ISSN: 1413-8131 (versão impressa) ISSN: 2358-9426 (versão eletrônica)

fragmentação e isolamento da área. Segundo Bierregaard Jr. et al. (1992), ambientes fragmentados atuam de forma negativa sob comunidades biológicas, reduzindo a biodiversidade local e podendo futuramente leva-las a extinção.

\section{Considerações finais}

Apesar de que outros métodos de captura de abelhas quando inseridos na metodologia de inventário resultam em um número maior de espécies na listagem final, a metodologia de coleta das abelhas em flores mostrou-se suficiente e foi confirmada pelos estimadores de riqueza utilizados no presente estudo.

A realização de outros inventários envolvendo a fauna de abelhas nas demais áreas de restinga no sul de Santa Catarina seria importante para o melhor entendimento da diversidade regional do grupo das abelhas, possibilitando a criação de medidas estratégicas para a preservação da apifauna. Sugere-se também a execução de futuros trabalhos afim de criar estratégias de manejo, como por exemplo, a implementação de possíveis corredores ecológicos na região estudada para interligar o fragmento em questão com outras áreas florestadas mais próximas.

\section{Agradecimentos}

Os autores agradecem à Universidade do Extremo Sul Catarinense (UNESC) pela bolsa de Iniciação Científica (PIBIC) concedida a dois autores deste artigo e aos revisores designados, pelas sugestões apresentadas.

\section{Referências}

ALBERTONI, E. F.; ESTEVES, F. A. Jurubatiba, uma restinga peculiar. Ciência Hoje, Rio de Janeiro, v. 25, n.148, p. 61-63, 1999.

AlBuQUERQUE, P. M. C; CAMARGO, J. M. F; MENDONÇA, J. A. C. Bee comunity of a beach dune ecosystem on Maranhão Island, Brazil. Brazilian Archives of Biology and Tecnology. v. 50, n. 6, p. 1005-1018, 2007.

ALVES-DOS-SANTOS, I. Bienen und melittophile Blütenpflanzen in der Küstenregion und im Atlantischen Regenwald von Rio Grande do Sul (Brasilien), mit einer Fallstudie zu Langzungenbienen und tristylen Wasserhyazinthen. 1996. 149 f. Tese (Doutorado em Ciências Naturais) - Universität Tübingen, Tübingen. 1996.

ALVES-DOS-SANTOS, I. Abelhas e plantas melíferas da Mata Atlântica, restinga e dunas do Litoral norte do Estado do Rio Grande do Sul. Revista Brasileira de 
Revista Tecnologia e Ambiente, v. 21, 2014, Criciúma, Santa Catarina. ISSN: 1413-8131 (versão impressa) ISSN: 2358-9426 (versão eletrônica)

Entomologia. v. 43, n. 3-4, p. 191-223, 1999.

ALVES-DOS-SANTOS, I. Comunidade, conservação e manejo: o caso dos polinizadores. Rev. Tecnologia e Ambiente. Criciúma, v.8, n.2, p. 35-57, 2002.

ALVES-DOS-SANTOS, I. Estudos sobre comunidades de abelhas no sul do Brasil e proposta para avaliação rápida da apifauna subtropical. Brazilian jornal of ecology, Rio Claro, Ano 11, nº 1-2, p. 53- 65, 2007.

ARAÚJO, D.S.D.; LACERDA, L.D. A natureza da restinga. Ciência Hoje. v. 6, n. 33, p. 42-48, 1987.

BENSUSAN, N. Conservação da biodiversidade: em áreas protegidas. Rio de Janeiro: Ed. FGV, 2006. 176p.

BEZ, M. Diversidade de abelhas, plantas visitadas e fenologia da floração em fragmento florestal urbano no município de Criciúma, Santa Catarina. 2009. $50 \mathrm{f}$. Monografia (Ciências Biológicas - Bacharelado) - Universidade do Extremo Sul Catarinense, Criciúma. 2009.

BIERREGAARD jr, R. O; LOVEJOY, T. E; KAPOS, V; SANTOS, A. A; HUTCHINGS, R. W. The biological dynamics of Tropical Rainforest fragments: A prospective comparison of fragments and continuous forest. BioScience. v. 42, n. 11, 1992.

BRASIL. 1999. Resolução do CONAMA n. 261, de 30 de junho de 1999. Define os parâmetros básicos para análise dos estágios sucessionais de vegetação de restinga para o Estado de Santa Catarina. Disponível em: <http://www.mma.gov.br/port/conama/res/res99/res26199.html>. Acesso em 03 nov. 2013.

BUZZI, Z. J. Entomologia didática. 4. Curitiba, PR: UFPR, 2005. 347 p.

CASCAES, M. A comunidade de abelhas (hymenoptera, apoidea) e flores visitadas em um fragmento de Mata Atlântica, no município de Maracajá, Santa Catarina. 2008. 59 f. Monografia (Ciências Biológicas - Bacharelado) - Universidade do Extremo Sul Catarinense, Criciúma. 2008.

COLWELL, R. K. Estimate S: Statistcal estimation of species richness and shared species from samples. Version 8. 2006.

CORDAZZO, C. V; SEELIGER, U. Composição e distribuição da vegetação nas dunas costeiras ao sul de Rio Grande (RS). Ciência e Cultura. v. 39, n.3, p. 321-324, 1997.

DORNELES, L. P. P; WAECHTER, J. L. Estrutura do componente arbóreo da floresta arenosa de restinga do Parque Nacional da Lagoa do Peixe, RS. Hoehnea. v. 31, n. 1, p. 61-71, 2004.

ENGEL, M. S. Classification of the bee tribe Augochlorini (Hymenoptera: Halictidae). American Museum of Natural History. v. 250, p. 1-90, 2000.

EPAGRI. Dados e Informações Biofísicas da Unidade de Planejamento Regional Litoral Sul Catarinense - UPR 8. Florianópolis, 2001.

FALKENBERG, D. B. Aspecto da flora e da vegetação secundária da Restinga de Santa Catarina, sul do Brasil. Insula. Florianópolis, n. 28, p. 1-30, 1999. 
Revista Tecnologia e Ambiente, v. 21, 2014, Criciúma, Santa Catarina. ISSN: 1413-8131 (versão impressa) ISSN: 2358-9426 (versão eletrônica)

GASPARIN, M; SEVEGNANI, L; DA ROSA, R. A.; SCHROEDER, E; OLIVEIRA, T. M; CRISTOFOLINI, K. Restinga assunto esquecido na escola. X Congresso de Ecologia do Brasil, São Lourenço-MG, 2011.

GONÇALVES, R. B.; MELO, G. A. R. A comunidade de abelhas (Hymenoptera, Apidae s.1.) em uma área restrita de campo natural no Parque Estadual de Vila Velha, Paraná:diversidade, fenologia e fontes florais de alimento. Revista Brasileira de Entomologia, Curitiba, v. 49, n. 4, p. 557-571, 2005.

GOTTSBERGER, G.; CAMARGO, J. M. F.; SILBERBAUER-GOTTSBERGUER, I. A bee-pollinated tropical community: the beach dune vegetation of Ilha de São Luís, Maranhão, Brazil. Botanische Jahrbüscher fur Systematik. v. 109, n. 4, p. 469-500, 1988.

HAMMER, Ø; HARPER, D.A.T; RYAN, P.D. PAST: Paleontological statistics software package for education and data analysis. Palaeontologia Electronica. v. 4, n. $1,2001$.

HARTER, B. Bienen und ihre Trachtpflanzen im Araukarien-Hochand von Rio Grande do Sul, mit Fallstudien zur Bestäubung vou Pionierpflazen. 1999. 185 f. Tese (Doutorado em Ciências Naturais) - Universität Tübingen, Tübingen. 1999.

IBM Corp. IBM SPSS Statistics for Windows, Version 20.0, Armonk, NY, 2011.

KAMKE, R; ZILLIKENS, A; STEINER, J. Species richness and seasonality of bees (Hymenoptera, Apoidea) in a restinga area in Santa Catarina, southern Brazil. Studies on Neotropical Fauna and Environment. v. 46, n. 1, p. 35-48, 2011.

KEVAN, P. G. Pollinators as bioindicators of the state of the environment: species, activity and diversity. Agriculture, Ecosystems and Environment. v. 74, p. 373-393, 1999.

KNEIP, L. M. Sambaquis na pré-história do Brasil. Ciência Hoje, v. 6, p33, p. 50-54. 1987.

KORTE, A; GASPER, A. L; KRUGER, A; SEVEGNANI, L. Composição florística e estrutura das Restingas em Santa Catarina. In: VIBRANS, A. C et al. Inventário Florístico de Santa Catarina. v. 4. Floresta Ombrófila Densa Blumenau: Edifurb. 2013. p. 285-309.

KRUG, C. A comunidade de abelhas (Hymenoptera, Apiformes) da Mata com Araucária em Porto União-SC e Abelhas visitantes florais da aboboreira (Cucurbita L.) em Santa Catarina, com notas sobre Peponapis fervens (Eucerini, Apidae). 2007. 120 f. Dissertação (Mestrado em Ciências Ambientais) - Universidade do Extremo Sul Catarinense, Criciúma. 2007.

KRUG, C; ALVES-DOS-SANTOS, I. O uso de diferentes métodos para amostragem da fauna de abelhas (Hymenoptera: Apoidea), um estudo em Floresta Ombrófila Mista em Santa Catarina. Neotropical Entomology. v. 37, n. 3, p. 265 - 278, 2008.

LEWINSOHN, T. M.; PRADO, P. K. L.; ALMEIDA, A. M. Inventários bióticos centrados em recursos: insetos fitófagos e plantas hospedeiras. In: DIAS, B. F. S.; GARAY, I. Conservação da biodiversidade em ecossistemas tropicais: Avanços conceituais de novas metodologias de avaliação e monitoramento. Petrópolis: Vozes, 2001. p. 174-189. 
Revista Tecnologia e Ambiente, v. 21, 2014, Criciúma, Santa Catarina. ISSN: 1413-8131 (versão impressa) ISSN: 2358-9426 (versão eletrônica)

MADEIRA-DA-SILVA, M. C; MARTINS, C. F. Abelhas (Hymenoptera, Apoidea: Apiformes) de uma área de restinga, Paraíba, Nordeste do Brasil: Abundância, diversidade e sazonalidade. Revista Nordestina de Biologia, v. 17, n. 1-2, p. 75-90, 2003.

MARTINS, C. F; CORTOPASSI-LAURINO, M; KOEDAM, D; IMPERATRIZFONSECA, V. L. Espécies arbóreas utilizadas para nidificação por abelhas sem ferrão na caatinga (Seridó, PB; Jõao Câmara, RN). Biota Neotropica. v. 4, n. 2, p. 1-8, 2004.

MELO, G.A.R; GONÇALVES, R.B. Higher-level bee classifications (Hymenoptera, Apoidea, Apidae sensu lato). Revista Brasileira de Zoologia, Curitiba, v. 22, p. 153159, 2005.

MENEZES, C. et al. Competição entre abelhas durante forrageamento em Schefflera arboricola (Hayata) Merr. Bioscience Journal, Uberlândia, v. 23, n. 1, p.63-69, 2007.

MICHENER, C. D. The bees of the world. 2. ed. Baltimore: Johns Hopkins University Press. 2007. 953p.

MOUGA, D. M. D. S. As comunidades de abelhas (Hymenoptera, Apoidea) em Mata Atlântica na região nordeste do Estado de Santa Catarina, Brasil. 2004. 253 f. Tese. Universidade de São Paulo, São Paulo. 2004.

MOUGA, D. M. D. S; NOBLE, C. F; BUSSMANN, D. B. G; KRUG, C. Bees ans plants in a transition area between Atlantic Rain Forest and Araucarian Forest in southern Brazil. Revue D Ecologie-La Terre Et La Vie, vol. 67, p. 313 - 327.2012.

MOURE, J. S; MELO, G. A. R; URBAN, D. Catalogue of Bees (Hymenoptera, Apoidea) in the Neotropical Region. Sociedade Brasileira de Entomologia, 2007. 1058 p.

NOGUEIRA-NETO, P. Vida e criação de abelhas indígenas sem ferrão. São Paulo: Editora Nogueirapis, 1997. 447 p.

OLIVEIRA, F. S; MENDONÇA, M. W. A; VIDIGAL, M. C. S; RÊGO, M. M. C; ALBUQUERQUE, P. M. C. Comunidade de abelhas (Hymenoptera, Apoidea) em ecossistema de dunas na Praia de Panaquatira, São José de Ribamar, Maranhão, Brasil.

Revista Brasileira de Entomologia. v. 54, n. 1, p.82-90, 2010.

OLLERTON, J; WINFREE, R; TARRANT, S. How many flowering plants are pollinated by animals? Oikos. v. 120, p. 321-326, 2011.

ORTOLAN, S. M L. S. Biocenótica em Apoidea (Hymenoptera) de áreas de macieira (Pyrus malus L.) em Lages, Santa Catarina, com notas comparativas e experimento preliminar de polinização com Plebeia emerina. Curitiba, PR: UFPR. p. 170. 1989.

PEREIRA, M. L. M; SOUZA, K. I. S; VIEIRA, C. V. Restingas: ser ou não ser, eis a questão. XIII Congresso da Associação Brasileira de Estudos do Quaternário ABEQUA, III Encontro do Quaternário Sulamericano. 2011.

RAVEN, P; EVERT, R; EICHHORN, S. Biologia Vegetal.7ed. Rio de Janeiro: Guanabara Koogan, 2007. 830 p.

REITZ, R. 1961. Vegetação da zona marítima de Santa Catarina. Sellowia. v. 13, n. 13, p. $17-115$. 
Revista Tecnologia e Ambiente, v. 21, 2014, Criciúma, Santa Catarina. ISSN: 1413-8131 (versão impressa) ISSN: 2358-9426 (versão eletrônica)

RICKLEFS, R. E. A Economia da Natureza. $6^{\text {a }}$ ed. Rio de Janeiro: Editora Guanabara Koogan, 2010.

ROSA 2011. Interações entre abelhas (hymenoptera, apidae) e plantas em área de regeneração natural em floresta ombrófila densa montana, no sul de santa catarina. 2011. 74 f. Monografia (Ciências Biológicas - Bacharelado) - Universidade do Extremo Sul Catarinense, Criciúma. 2011.

ROUBICK, D. W. Ecology and natural history of tropical bees. New York: Cambridge University Press, 1989. 514 p.

SAKAGAMI, S. F; LAROCA, S; MOURE, J. S. Wild bees biocenotics in São José dos Pinhais (PR), South Brazil - preliminary report. Journal of the Faculty of Science Hokkaido University (Ser. 6, Zoology). n. 19, p. 253-291, 1967.

SAKAGAMI, S. F; LAROCA, S. Relative abundance, phenology and flower visits of Apid bees in eastern Paraná, southern Brazil. Kontyu, Tokyo. v.39, n.3, 217-230 p, 1971.

SANTA CATARINA. Disponível em: <http://www.sc.gov.br>. Acesso em 11 de mar. 2013.

SCHLINDWEIN, C. Frequent oligolectic characterizing a diverse bee-plant community in a xerophytic bushland of subtropical Brazil. Studies on Neotropical Fauna and Environment. v. 33, p. 46 -59. 1998.

SCHWARTZ-FILHO, D. L; LAROCA, S. A comunidade de abelhas silvestres (Hymenoptera, Apoidea) da Ilha das Cobras (Paraná, Brasil): aspectos ecológicos e biogeográficos. Acta Biológica Paranaense. v. 28, p. 19-108, 1999.

SILVA, M. Abelhas e plantas melíferas da zona rural dos municípios de Cocal do Sul, Criciúma e Nova Veneza, situados na região carbonífera no sul do estado de Santa Catarina. 2005. 110 f. Dissertação (Mestrado em Ciências Ambientais) Universidade do Extremo Sul Catarinense, Criciúma. 2005.

SILVEIRA, F. A; MELO, G. A. R; ALMEIDA, E. A. B. Abelhas brasileiras: sistemática e identificação. Belo Horizonte: Fundação Araucária, 2002. 253 p.

SIMINSKI, A; FANTINI, A. F. Classificação da mata atlântica do litoral catarinense em estádios sucessionais: ajustando a lei ao ecossistema. Floresta e ambiente. v. 11, n.2, p. $20-25,2004$.

STEINER, J; HARTER-MARQUES, B; ZILLIKENS, A; FEJA, E. P. Bees of Santa Catarina Island, Brazil - a first survey and checklist (Insecta: Apoidea). Zootaxa. v, 1220. p. 1-18, 2006.

TRUYLIO, B; HARTER-MARQUES, B. A comunidade de abelhas (Hymenoptera, Apoidea) em áreas florestais do Parque Estadual de Itapuã (Viamão, RS): diversidade, abundância relativa e atividade sazonal. Iheringia, Série Zoologia. v. 97, p.392-399, 2007.

VIANA, B. F. A Comunidade de Abelhas (Hymenoptera: Apoidea) das Dunas Interiores do Rio São Francisco, Bahia, Brasil. Anais da Sociedade Entomológica do Brasil. v. 28, n. 4, p. 635 - 645. 1999.

VIANA, B. F; ALVES-DOS-SANTOS, I. Bee Diversity of the Coastal Sand Dunes of 
Brazil. In: KEVAN, P; IMPERATRIZ-FONSECA, V. L. Pollinating Bees - The Conservation Link Between Agriculture and Nature - Ministry of Environment, Brasília. p. 135-153. 2002.

VIANA, B. F; KLEINERT, A. M. P. A community of flower-visiting bees (Hymenoptera: Apoidea) in the coastal sand dunes of northeastern Brazil. Biota Neotropica. v.5, n.2, p. 1-13, 2005.

WILMS, W; IMPERATRIZ-FONSECA, V. L; ENGELS, W. Resource partioning between highly eusocial bees and possible impact of the introduced Africanized honey bee on native stingless bees in the brasilian atlantic rainforest. Studies on Neotropical Fauna and Environment. v. 31, p. 137 - 151. 1996.

WITTMANN, D; HOFFMAN, M. Bees of Rio Grande so Sul, southern Brazil (Insecta, Hymenoptera, Apoidea). Iheringia, Série Zoologia., Porto Alegre. v. 70, p. 17 43.1989.

ZANELLA, C. V; SCHWARTZ-FILHO, D. L; LAROCA, S. Tropical bee island. Biogeography: Diversity and abundance patterns. Biogeographica. v. 74, n. 3, p. $103-$ 115, 1998.

ZAR, J. H. Biostatistical Analysis. 4. ed. New Jersey: Prentice Hall, 1999. 